\title{
A CONSTRUCTION OF A BASE FOR THE M FOLD TENSOR PRODUCT OF A BRANCH SPACE
}

\author{
ROHAN HEMASINHA \\ The University of West Florida \\ Pensacola, Florida 32514 \\ (Received August 3, 1987)
}

ABSTRACT. Let $E$ be a Banach space with Schauder base $\left(x_{n}\right)_{n}$. Let $\stackrel{m}{\bullet} E$ denote the completion of the $m$ fold tensor product with respect to a reasonable cross norm $\alpha$. We show that the set $\left\{x_{1} \bullet \ldots \bullet x_{1_{m}}: x_{1_{j}} \varepsilon\left(x_{n}\right)_{n}\right\}$ can be enumerated so that for each positive integer $k$, the first $k^{m^{m}}$ terms are precisely all the elements of the form

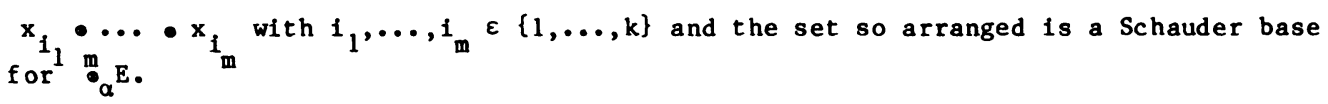
KEYWORDS AND PHRASES. m fold tensor product, profective, injective and reasonable cross norms, Schauder bases.

1980 AMS SUBJECT CLASSIFICATION CODE. 46B15, 46M05.

\section{INTRODUCTION.}

Given a Banach space $E$, we will denote by $\mathrm{m}_{\mathrm{E}}$ the $\mathrm{m}$ fold algebraic tensor product and by ${ }_{\alpha} \mathrm{E}$ its completion with respect to reasonable norm $\alpha_{\text {. If }}\left(x_{n}\right)_{n}$ is a Schauder base for $E$ then $\bullet \cdot\left(x_{n}\right)$ will denote the set

$$
\left\{x_{1_{1}} \bullet \ldots \bullet x_{1_{m}}: x_{1_{j}} \varepsilon\left(x_{n}\right), 1<j<m\right\}
$$

For $m>2$ the projective (injective) tensor norm on $\stackrel{m}{E}_{E}$ will be denoted by $\gamma_{m}\left(\lambda_{m}\right)$. If $m=2$ this norm will be denoted by $\gamma(\lambda)$.

In [1], [4] it is shown that if $E_{1}, E_{2}$ are Banach spaces with Schauder bases $\left(x_{n}\right)_{n},\left(y_{n}\right)_{n}$ then for any reasonable norm $\alpha$, the space $E_{1} \bullet_{\alpha} E_{2}$ has a Schauder base $\left(z_{n}\right)$ with the following properties.

(1) $\left(z_{n}\right)_{n}$ is obtained by enumerating the set $\left(x_{n}\right)_{n} \cdot\left(y_{n}\right)_{n}$;

(2) the enumeration is such that for any positive integer $k$, the first $k^{2}$ terms are precisely all elements of the form

$$
x_{1} \cdot y_{1}, \quad 1_{1}, i_{2} \in\{1, \ldots, k\}
$$


In this paper we show that for any positive integer $m$, the space $\bullet_{\alpha} E$ has a Schauder base obtained by enumerating $\stackrel{m}{\bullet}\left(x_{n}\right)_{n}$ in such a way that for any positive integer $k$, the first $k^{m}$ terms are precisely all elements of the form

$$
x_{i_{1}} \bullet \ldots \bullet x_{i_{m}} \text { with } i_{1}, \ldots, i_{m} \varepsilon\{1, \ldots, k\}
$$

Our proof is via an induction argument and in a forthcoming paper we utilize this construction to derive some properties of the symmetric tensor algebra of a Banach space. Note that even for the case $m=3$, iteration to $\left(E \bullet_{\gamma} E\right) \cdot{ }_{Y} E$ of the enumerating scheme described in [1] will not yield a base with the above mentioned properties.

For definitions and terminology regarding Schauder bases, tensor products and tesnor norms we refer the reader to [4], [3], and [2].

We denote the following property (*) of the projective tensor norm. For $\mathrm{m}>2$ let $u$ be an element of $\bullet_{\mathrm{E}}$. It can easily be shown that

$$
\gamma(u)=\gamma_{m}(u)
$$

where $\gamma(u)$ is the projective norm of $u$ when $u$ is considered as an element of $F$ - E with $F={ }^{m-1} E$ being endowed with the norm $\gamma_{m-1}$. We shall make use of this fact subsequent ly.

We now state our theorem.

THEOREM. Let $\left(x_{n}, f_{n}\right), x_{n} \in E, f_{n} \in E^{\prime}$ be biorthogonal system such that $\left(x_{n}\right)_{n}$ is a Schauder base for E. Let $\alpha$ be a reasonable norm on $\bullet_{E}$. Then there exists for ${ }_{a}^{m} E$ a biorthogonal system $\left(z_{n}, g_{n}\right)$ with the following properties.

(1) As $\operatorname{sets}\left[z_{n}: n \varepsilon N\right\}=\stackrel{m}{\bullet}\left(x_{n}\right)_{n}$

$$
\left[g_{n}: n \in N\right\}=m_{\left(f_{n}\right)} \text {. }
$$

(2) The enumeration of $\left(z_{n}, g_{n}\right)$ is such that for each positive integer $k$ the first $k^{m}$ terms are precisely all the tensors of the form $x_{1_{1}} \bullet \cdots \bullet x_{1_{m}}$,

$\mathrm{f}_{\mathrm{i}_{1}} \bullet \ldots \bullet \mathrm{f}_{\mathrm{i}_{\mathrm{m}}}$ with $\mathrm{i}_{1}, \ldots, \mathrm{i}_{\mathrm{m}} \in\{1, \ldots, \mathrm{k}\}$.

(3) The sequence $\left(z_{n}\right)_{n}$ is a Schauder base for $\bullet_{\alpha}^{m}$.

PROOF. It is known that [2] $\lambda_{\mathrm{m}}<\alpha<\gamma_{\mathrm{m}}$ for any reasonable norm $\alpha$. Also ${ }^{m} E^{\prime} \subseteq\left(\bullet^{m} \lambda_{m} E\right)^{\prime} \cdot m$ Thus any enumeration of $\bullet_{m}^{m}\left(x_{n}\right)_{n}$ together with the corresponding enumeration of ${ }^{\circ}\left(f_{n}\right)_{n}$ yields a biorthogonal system for ${ }^{m} E$. It is easily shown that the linear span of ${ }^{m}\left(x_{n}\right)_{n}$ is dense in ${ }^{m} E$ and we shall first establish the assertions of the theorem for $\alpha=\gamma_{m}$.

Now for $m=2$ the base constructed in [1] has the stated properties.

Suppose that a biorthogonal system $\left(z_{n}, g_{n}\right)$ with the stated properties can be constructed for $\bullet_{\mathrm{m}}^{\mathrm{m}} \mathrm{E}$. 
Consider the sequence $\left(w_{n}\right)_{n}$ in ${ }^{m+1}$ E defined by the scheme in the accompanying figure. For each positive integer $k$, the terms

$$
\left.w_{k}{ }^{m+1}+1, w_{k}^{m+1}+2, \cdots, w_{(k+1}\right)^{m+1}
$$

are described by the tensor beneath each term.

\section{Figure 1}

$k$, a positive integer; $\ell$ an integer $0<\ell<k$

\begin{tabular}{|c|c|c|}
\hline $\mathrm{w}_{\mathrm{k}^{\mathrm{m}+1}+1}$ & $\mathrm{w}_{\mathrm{k}^{\mathrm{m}}}^{\mathrm{m}+1}+2$ & ${ }^{\mathrm{w}}(\mathrm{k}+1)^{\mathrm{m}}+\mathrm{k}^{\mathrm{m}}(\mathrm{k}-1)$ \\
\hline$x_{1} \bullet z_{k} m_{+1}$ & $x_{1} \cdot z_{k} m_{+2}$ & $x_{1} \cdot z_{(k+1)^{m}}$ \\
\hline${ }^{\mathrm{w}}(\mathrm{k}+1)^{\mathrm{m}}+\mathrm{k}^{\mathrm{m}}(\mathrm{k}-1)+1$, & ${ }^{\mathrm{w}}(\mathrm{k}+1)^{\mathrm{m}}+\mathrm{k}^{\mathrm{m}}(\mathrm{k}-1)+2$, & ${ }^{w} 2(k+1)^{m}+k^{m}(k-2)$ \\
\hline$x_{2} \odot z_{k} m_{1}$ & $x_{2} \cdot z_{k}{ }^{m}+2$ & $x_{2} \cdot z_{(k+1)^{m}}^{m}$ \\
\hline • & • & • \\
\hline$\dot{.}$ & $\dot{.}$ & $\dot{.}$ \\
\hline${ }^{\mathrm{w}} \ell(\mathrm{k}+1)^{\mathrm{m}}+\mathrm{k}^{\mathrm{m}}(\mathrm{k}-\ell)+1$, & $\ldots \ldots \ldots \ldots \ldots \ldots$ & ${ }^{\mathrm{w}}(\ell+1)(\mathrm{k}+1)^{\mathrm{m}}+\mathrm{k}^{\mathrm{m}}(\mathrm{k}-(\ell+1))$ \\
\hline$x_{\ell}+1 \cdot z_{k} m_{+1}$ & $\ldots \ldots \ldots \ldots \ldots$ & $\mathrm{x}_{\ell+1} \cdot \mathrm{z}_{(\mathrm{k}+1)^{\mathrm{m}}}$ \\
\hline${ }^{\mathrm{w}}(\mathrm{k}-1)(\mathrm{k}+1)^{\mathrm{m}}+\mathrm{k}^{\mathrm{m}}+1$, & $\cdots \ldots \ldots \ldots \ldots$ & $w_{k}(k+1)^{m}$ \\
\hline$x_{k} \cdot z_{k} m_{+1}$ & $\cdots \ldots \ldots \ldots \ldots$ & $x_{k} \cdot z_{(k+1)^{m}}$ \\
\hline $\mathrm{w}_{\mathrm{k}}(\mathrm{k}+1)^{\mathrm{m}}+1$ & $w_{k}(k+1)^{m}+2$ & ${ }^{w}(k+1)^{m+1}$ \\
\hline
\end{tabular}

In the preceding tableau each of the first $k$ rows contains $(k+1)^{m}-k^{m}$ entries. The last row has $(k+1)^{m}$ entries, and so altogether we have exhibited $k\left((k+1)^{m}-k^{m}\right)+(k+1)^{m}=(k+1)^{m+1}-k^{m+1}$ entries.

The sequence $\left(h_{n}\right)$ is obtained by enumerating the set $\left\{g_{p} \bullet f_{q} \mid g_{p} \varepsilon\left(g_{n}\right), f_{p} \varepsilon\left(f_{n}\right)\right\}$ according to the same scheme. 
In view of the inductive hypothesis the system $\left(w_{h}, h_{n}\right)$ is a biorthogonal system with the property that the first $k^{m+1}$ terms are all of the form

$$
x_{1} \bullet \cdots \cdot x_{i_{m+1}}, f_{1_{1}} \bullet \cdots \cdot f_{i_{m+1}}
$$

with $i_{1}, \ldots, i_{m+1} \in\{1, \ldots, k\}$.

Let $S_{n}, T_{n}, W_{n}$ respectively denote the $n^{\text {th }}$ partial sum operators of the systems $\left(x_{n}, f_{n}\right),\left(z_{n}, g_{n}\right)$ and $\left(w_{n}, h_{n}\right)$.

Since $\left(x_{n}\right),\left(z_{n}\right)$ are bases the sequences $\left(f_{n}\right),\left(T_{n}\right)$ are bounded in operator norro by some constant $M$. To show that $\left(w_{n}\right)$ is a base it thus suffices to show that for some positive $M^{\prime},\left\|W_{n}\right\|<M^{\prime}$ for all $n([4]$, p. 25).

Now, given a positive integer $n$ the defining scheme for ( $\left.w_{f}\right)$ (see fig. 1) shows that $W_{n}$ can be expressed as sums of tensor products of the operators $S_{m}, T_{m}$ and $f_{m}$.

Indeed, let us consider the three possible cases.

Case 1. If $n=k^{m+l}$ for some positive integer $k$ then

$$
W_{n}=\stackrel{m+1}{\bullet S_{k}} \text {, the m+l fold tensor product of } S_{k} \text {. }
$$

Case 2. If $\mathrm{n}=\ell(\mathrm{k}+1)^{\mathrm{m}}+\mathrm{k}^{\mathrm{m}}(\mathrm{k}-\ell)+\mathrm{r}$, with $0<\ell<\mathrm{k}$ and

$$
\begin{aligned}
& 1<r<(k+1)^{m}-k^{m} \text { then } \\
& W_{n}={ }^{m+1} S_{k}+\left(T(k+1) m-T_{k} m\right) \cdot S_{\ell}+\left(T_{k} m_{+r}-T_{k}^{m}\right) \cdot f_{\ell+1} \cdot \\
& \text { Case 3. If } n=k(k+1)^{m}+r \text { with } 1<r<(k+1)^{m} \text { then } \\
& W_{n}=\stackrel{m+1}{\bullet} S_{k}+\left(T(k+1) m-T_{k} m\right) \cdot S_{k}+T_{r} \bullet f_{k+1}
\end{aligned}
$$

In $u$ is in ${ }^{m+l} E$ then property (*) together with the fact that $\gamma_{m+1}$ is reasonable yields the following inequalities

(i) $\gamma_{m+1}\left({ }^{m+1} s_{k}(u)\right)<\left\|s_{k}\right\|^{m+1} \gamma_{m+1}(u)$

(2) $\left.\gamma_{m+1}\left((T, k+1) m-T_{k} m\right) \cdot S_{\ell}(u)\right)$

$$
<\left\|T T_{(k+1)} m-T_{k} m\right\| S_{\ell} \| \gamma_{m+1}(u)
$$

(3) $\gamma_{m+1}\left(\left(T_{k} m_{+r}-T_{k} m\right) \cdot f_{\ell+1}(u)\right)$

$$
<\left\|T_{k} m_{+r}-T_{k} m\right\|\left\|f_{\ell+1}\right\| \gamma_{m+1}(u) .
$$


We utilized (*) in deriving (2) and (3). Hence,

$$
\gamma_{m+1}\left(W_{n}(u)\right)<\left(M^{m+1}+2 M^{2}+2 M^{2}\right) \gamma_{m+1}(u)
$$

in all three cases. It now follows froin the uniform boundedness principle that $\left(\left\|w_{n}\right\|\right)$ is bounded.

To complete the proof let us recall that if $\alpha$ is a reasonable norm then $\lambda_{m}<\alpha<\gamma_{m}$. Furthermore, $\stackrel{\bullet}{\alpha}_{\alpha} \mathrm{E}$ is the completion of $\mathrm{m}_{\mathrm{E}}$ with respect to $\alpha$. Consequently for all such $\alpha$, the system $\left(z_{n}, g_{n}\right)$ is a complete biorthogonal system whose sequence of partial sums $T_{n}$ is pointwise bounded and hence bounded in operator norm. This means that $\left(z_{n}\right)_{n}$ is a Schauder base for ${ }_{\alpha} E$.

CONCLUDING REMARKS •

It would be interesting to find out whether the base for ${ }_{\gamma_{m}} E$ obtained by iteration of the process in [1] is equivalent to the base described in tnis paper. We bope to investigate this problem in a future paper.

\section{REFERENCES}

1. GELBAUM, B.R. and GIL DE LA MADRID, J. Bases for Tensor Products of Banach Spaces, Pacific J. Math. $\underline{11}$ (1961), pp. 1281-1286.

2. CIGleR, J., LOSERT, V. and MICHOR, P. Banach Modules and Functors on Categorles of Banach Spaces. Lecture Notes in Pure and Applied Mathematics, Marcel Dekker, Inc., (1979).

3. SCHATTEN, R. A Theory of Cross Spaces. Annals of Mathematics Studies, 26, Princeton University Press, (1950).

4. Singer, I. Bases for Banach Spaces, Vol. 1. A Series of Comprehensive Studies in Mathematics, 154. Springer-Verlag, (1970). 


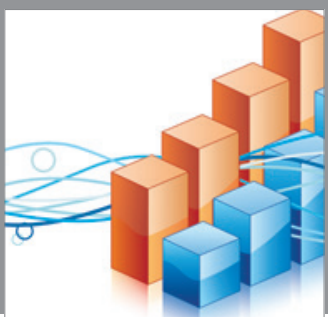

Advances in

Operations Research

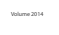

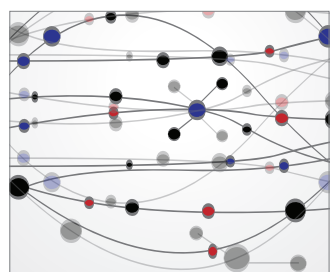

\section{The Scientific} World Journal
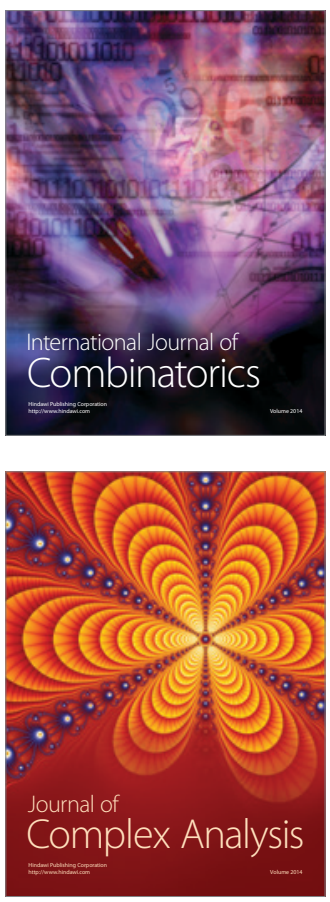

International Journal of

Mathematics and

Mathematical

Sciences
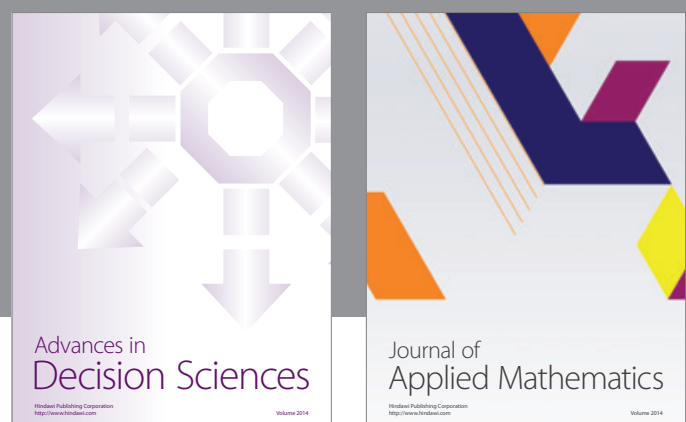

Journal of

Applied Mathematics
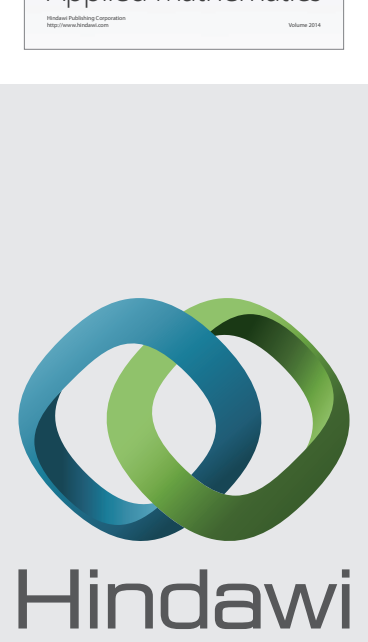

Submit your manuscripts at http://www.hindawi.com
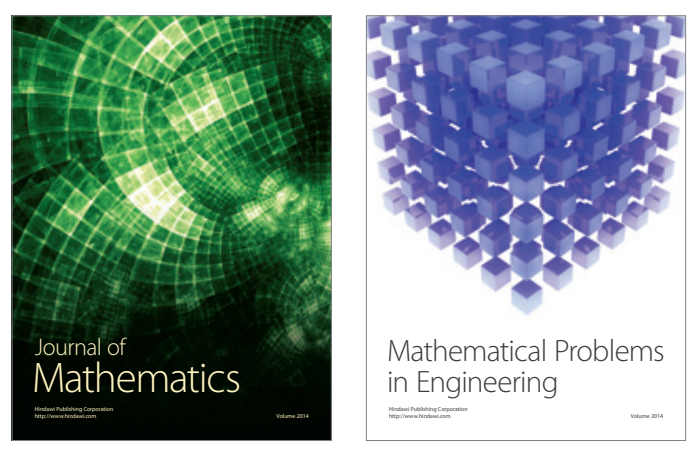

Mathematical Problems in Engineering
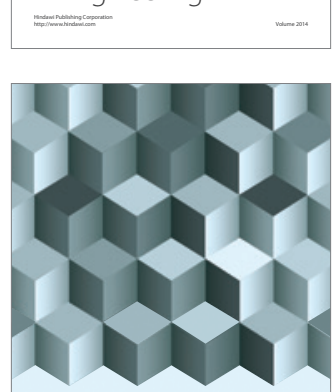

Journal of

Function Spaces
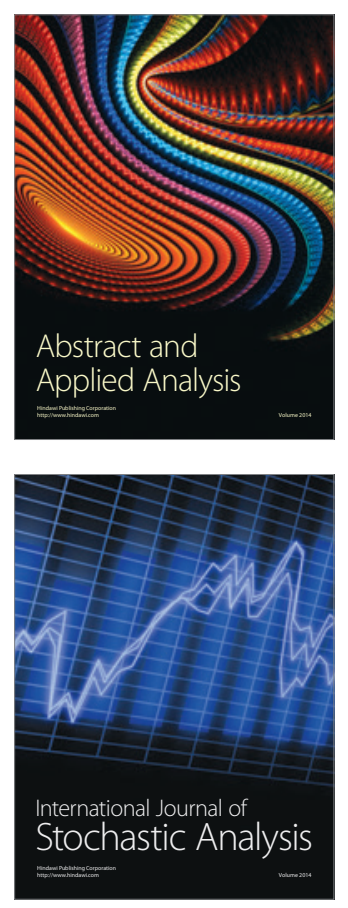

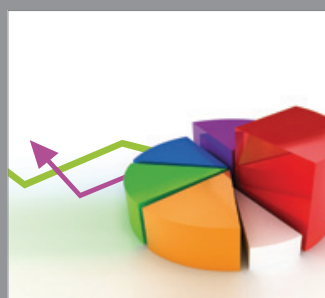

ournal of

Probability and Statistics

Promensencen
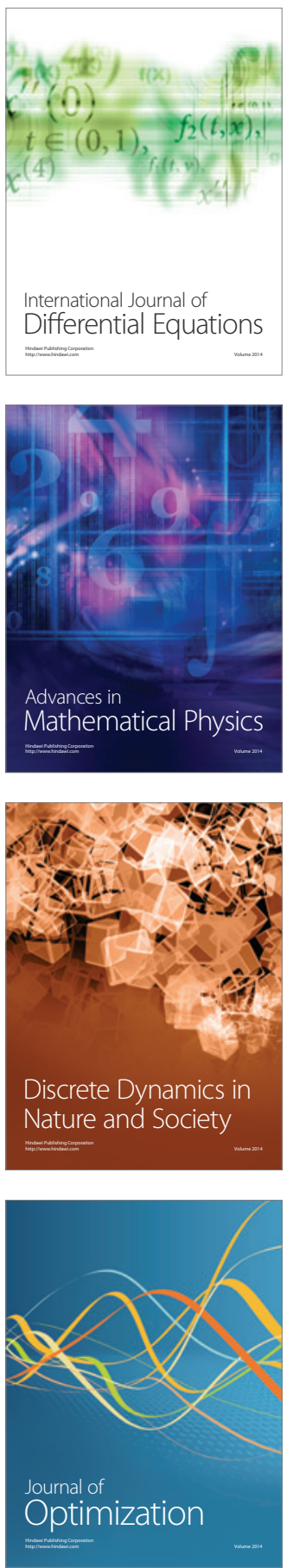